\title{
Considering the base rates of low performance in cognitively healthy older adults improves the accuracy to identify neurocognitive impairment with the Consortium to Establish a Registry for Alzheimer's Disease-Neuropsychological Assessment Battery (CERAD-NAB)
}

\author{
Panagiota Mistridis · Simone C. Egli $\cdot$ Grant L. Iverson • \\ Manfred Berres • Klaus Willmes • Kathleen A. Welsh-Bohmer • \\ Andreas U. Monsch \\ Received: 24 June 2014 / Accepted: 19 December 2014 / Published online: 3 January 2015 \\ (C) The Author(s) 2015. This article is published with open access at Springerlink.com
}

\begin{abstract}
It is common for some healthy older adults to obtain low test scores when a battery of neuropsychological tests is administered, which increases the risk of the clinician misdiagnosing cognitive impairment. Thus, base rates of healthy individuals' low scores are required to more accurately interpret neuropsychological results. At present, this information is not available for the German version of the Consortium to Establish a Registry for Alzheimer's Disease-Neuropsychological Assessment Battery (CERAD-NAB), a frequently used battery in the USA and in German-speaking Europe. This study aimed to determine the base rates of low scores for the CERAD-NAB and to tabulate a summary figure of cut-off scores and numbers of low scores to aid in clinical decision making. The base rates of low scores on the ten German CERAD-NAB subscores
\end{abstract}

Electronic supplementary material The online version of this article (doi:10.1007/s00406-014-0571-z) contains supplementary material, which is available to authorized users.

P. Mistridis · S. C. Egli · A. U. Monsch $(\bowtie)$

Memory Clinic, Felix Platter Hospital, University Center

for Medicine of Aging Basel, Schanzenstrasse 55,

4031 Basel, Switzerland

e-mail: Andreas.Monsch@unibas.ch

P. Mistridis · S. C. Egli · A. U. Monsch

Department of Psychology, University of Basel, Missionsstrasse

60/62, 4055 Basel, Switzerland

\section{G. L. Iverson}

Department of Physical Medicine and Rehabilitation, Harvard

Medical School, Boston, MA 02114, USA

G. L. Iverson

Red Sox Foundation and Massachusetts General Hospital Home

Base Program, Boston, MA 02114, USA were calculated from the German CERAD-NAB normative sample $(N=1,081)$ using six different cut-off scores (i.e., 1st, 2.5th, 7th, 10th, 16th, and 25th percentile). Results indicate that high percentages of one or more "abnormal" scores were obtained, irrespective of the cut-off criterion. For example, $60.6 \%$ of the normative sample obtained one or more scores at or below the 10th percentile. These findings illustrate the importance of considering the prevalence of low scores in healthy individuals. The summary figure of CERAD-NAB base rates is an important supplement for test interpretation and can be used to improve the diagnostic accuracy of neurocognitive disorders.

Keywords Neuropsychology $\cdot$ Normal aging · Diagnosis $\cdot$ Neurocognitive disorders $\cdot$ Dementia . Mild cognitive impairment

M. Berres

Department of Mathematics and Technology, University of Applied Sciences Koblenz, Joseph-Rovan-Allee 2,

53424 Remagen, Germany

K. Willmes

Section Neuropsychology, Department of Neurology, RWTH

Aachen University, Pauwelsstraße 30, 52074 Aachen, Germany

K. A. Welsh-Bohmer

Joseph and Kathleen Bryan Alzheimer's Disease Center,

Duke University, 2200W Main Street, Suite A200, Durham,

NC 27705, USA 


\section{Introduction}

When administered a battery of neuropsychological tests, some healthy older adults obtain low scores (e.g., [1-6]). Low test scores, especially low memory scores, obtained by healthy older adults might be interpreted as an indication of cognitive deterioration and lead to a false-positive diagnosis of mild cognitive impairment (MCI). Thus, to evaluate test results accurately and safeguard against misinterpretation of abnormal test performance, knowledge about base rates of low scores in healthy older adults compared to true pathological performance is of critical importance.

Cognitive deficits, particularly in episodic memory, represent a hallmark of Alzheimer's disease (AD) dementia and are known to be detectable in prodromal stages, such as MCI [7-9]. However, given that healthy older adults may also show a gradual but clinically insignificant cognitive decline over time [10,11], these cognitive changes may be very subtle, such that in clinical practice detecting true cognitive impairment remains challenging [8]. Thus, to obtain an accurate understanding of an individual's cognitive functioning for a reliable and valid diagnosis, it is very important to differentiate normal changes with cognitive aging from cognitive changes that go beyond normal aging.

At present, however, no universally accepted and empirically validated psychometric criteria exist to define cognitive impairment $[12,13]$. Cognitive deterioration is broadly defined in clinical practice, and different cut-off scores are used to define impairment. Thus, when assessing cognitive impairment, some methodological issues have to be considered. When administering only one neuropsychological test, the number of individuals being within the lower tail of the Gaussian distribution will depend on the chosen cutoff score (e.g., when using the 7th percentile as the critical cut-off score, by definition, $7 \%$ of cognitively healthy individuals would be erroneously classified as impaired). However, clinicians usually do not rely on a single test score, but on the patients' performance on multiple tests when assessing cognition. The prevalence of low scores will then be considerably higher when the number of tests administered increases, compared to single test interpretation $[1,12]$. Additionally, tests in a neuropsychological battery commonly show substantial intercorrelations and are therefore not independent, such that a non-consideration of this fact results in inaccurate assumptions (i.e., over- or underestimation) of neuropsychological test results. Thus, the number of tests in a test battery and the cut-off criterion applied affect the number of low scores: that is, as more tests are administered and less stringent [e.g., 25th percentile $(z=-0.67)]$ cut-off scores are applied, then more "abnormal" scores will be obtained by healthy individuals $[12,14,15]$. Although the recommended criterion for low performance associated with $\mathrm{MCI}$ is 1.5 standard deviations
(SD) below the mean of adjusted normative data [16], other cut-off scores ranging from 1.0 to 2.0 SD below the mean have been proposed by the National Institute on Aging and the Alzheimer's Association [17] or the fifth edition of the American Psychiatric Association's Diagnostic and Statistical Manual of Mental Disorders (DSM-5; [18]). However, the applied cut-off score needs to be considered carefully because the cut-off score selected substantially affects the rate of false-positive low scores [12, 19]. In addition, beside such psychometrical arguments, personal or situational factors may affect the prevalence of low scores (see [20]). For example, some people will be located at the lowest (and highest) tail of the Gaussian distribution, i.e., these individuals have always performed low in the past and will also show weak performances in the future, although they are healthy. Longstanding weaknesses in certain cognitive areas may also account for low scores as well as situational factors such as the personal (daily) condition (poor sleep quality, fatigue, low motivation, attentional distraction, discomfort during testing or tension, suffering from pain, bad emotional status, difficulties to adapt to test situation, etc.). Another source might consist of measurement errors such as misunderstandings of test instructions.

The presence of low scores in healthy older adults is a well-known phenomenon and a relevant issue for clinicians and researchers to consider, especially in order to safeguard against misinterpretation of some "abnormal" scores. However, this concept still remains relatively poorly understood and has certainly not yet been implemented in its full significance in everyday clinical practice. Low scores are a common feature in any battery of tests and have already been reported by several research groups for different neuropsychological tests or test batteries (e.g., [2]). However, at present this information is not available for the German version of the Consortium to Establish a Registry for Alzheimer's Disease-Neuropsychological Assessment Battery (CERAD-NAB), a well-established and very commonly used battery in German-speaking Europe and the USA to assess mature individuals with potential neurocognitive disorders $[21,22]$. The CERAD-NAB yields ten standardized $z$-scores and is useful in discriminating between healthy older adults and patients with incipient $\mathrm{AD}$ or $\mathrm{AD}$ dementia [21, 23, 24]. Incorporating the base rates of low scores into clinical practice might reduce false-positive diagnoses that may cause anxiety and distress in both the affected individuals and their families, as well as reduce false-negative diagnoses that would prevent patients from receiving and profiting from early therapeutic interventions.

The objective of the present study was to determine base rates of low cognitive scores in healthy older adults on the CERAD-NAB using empirical data from an assessment of a large normative sample of healthy older participants. To account for the well-known influence of demographic 
variables, we used standardized $z$-scores adjusted for age, gender, and education [25] in all analyses. The base rates of low scores in the normative sample were calculated for multiple cut-off scores for the entire battery. In a further step, we provide a summary figure that tabulates the number of low CERAD scores for six cut-off scores (i.e., 1st, 2.5th, 7th, 10th, 16th, and 25th percentile) to aid clinicians to diagnose and differentiate between normal and abnormal performance. To examine the usefulness of this summary figure in identifying individuals at risk for cognitive deterioration at a very early stage, baseline performance of initially healthy participants who developed AD dementia several years later was compared with participants who remained healthy.

\section{Methods}

\section{Participants}

The study sample consisted of 1,099 healthy older participants from the BASEL study (BAsel Study on the ELderly) aiming to investigate preclinical cognitive markers of $A D$ (see $[26,27]$ for details) and who constituted the reference (i.e., the normative) sample for the German version of the CERAD-NAB [28]. Baseline testing was conducted between 1997 and 2001. A detailed description of the biannual follow-up assessments within the BASEL study is described elsewhere [26]. Eighteen participants were excluded from the baseline sample because a detailed review of the charts at follow-up revealed that these individuals should have been excluded based on medical exclusion criteria [i.e., prostate carcinoma $(n=5)$, use of psychoactive substances $(n=3)$, loss of consciousness of more than $5 \min (n=2)$, cardiac dysfunction/aortic stenosis $(n=2)$, sensory deficits $(n=2)$, history of high temperature episode $(n=2)$, temporal arteritis $(n=1)$, or chronic pain $(n=1)]$. Thus, the final sample consisted of $1,081 \mathrm{Ger}-$ man-speaking healthy normal participants (407 women/674 men). Age ranged from 49 to 92 years $(M=68.6$ years; $\mathrm{SD}=7.8$ years), and participants had a mean educational level of 12.5 years $(\mathrm{SD}=3.0$ years; range 7-20 years). Mini-Mental State Examination (MMSE; [29]) test scores ranged from 24 to 30 , with a mean of 28.9 ( $\mathrm{SD}=1.1)$. To ensure healthy baseline cognitive status of all participants, the following procedure to establish the gold standard was applied: All participants were interviewed with a comprehensive medical questionnaire and were only included in the analyses, when they were cognitively healthy. Participants were excluded if they fulfilled any of the following criteria (see also [27]): (1) severe hearing, visual, or verbal deficits that interfered with the administration of neuropsychological testing; (2) severe motor deficits that interfered with everyday life; (3) severe systemic diseases (e.g., severe cardiac, renal, or liver dysfunctions, or severe endocrinological, or gastrointestinal diseases); (4) chronic pain; (5) major psychiatric disorders according to the fourth edition of the DSM (DSM-IV; [30]); (6) current use of psychoactive substances; (7) current or past diseases of the central nervous system; (8) cerebrovascular diseases (e.g., stroke, transient ischemic attack); (9) generalized atherosclerosis; and (10) diseases or events during life that could have negatively affected the central nervous system (e.g., head trauma with loss of consciousness $>5 \mathrm{~min}$, alcohol abuse, or general anesthesia within the last 3 months). In addition, if a participant exhibited low cognitive performance (i.e., a $z$-score $\leq-1.96$ in more than one of 11 CERAD-NAB variables, including the MMSE [29] (see [27] for details) and their spouses (or another family member) reported a decline of the participant's cognitive functions, the participant was excluded from the normative sample.

Additionally, two subsamples of this normative sample were considered to examine potential differences in the number of low scores (see "Analyses" section) already at this stage of normalcy between participants who later developed $\mathrm{AD}$ dementia (NC-AD) and participants who remained cognitively healthy (NC-NC). Even a marginal difference in the number of low scores between these two samples would corroborate the importance to take base rates into account. The NC-NC subsample consisted of 26 participants who remained healthy during the whole observation time and who were matched for age ( \pm 5 years), gender (exact), and education ( \pm 5 years) to the 26 participants who were healthy at baseline, but developed AD dementia years later. In addition, each NC-NC participant needed to be in the study for at least as long as his/her NC-AD counterpart. AD was diagnosed by the Memory Clinic Basel according to the National Institute of Neurological and Communicative Disorders and Stroke and the Alzheimer's Disease-Related Disorders Association (NINCDSADRDA) criteria [31] and the DSM-IV [30] after referral from study coordinators due to participant-or informantbased concerns about cognitive deterioration or objective cognitive decline. Diagnosis was based on additional neuropsychological results, magnetic resonance imaging, medical and neurological examination as well as blood and serum analyses [32]. The two subgroups were comparable with respect to age, years of education, gender distribution, and MMSE score, but differed with respect to observation time such that $\mathrm{NC}-\mathrm{NC}$ participants were significantly longer in the study compared to NC-AD participants (see Table 1). AD dementia was diagnosed in the NC-AD group after approximately 8 years $(M=8.5$ years, range $3.2-$ 13.3 years). The study was approved by the Ethics Committee of both Basel (Switzerland) and was performed in accordance with the ethical standards laid down in the 1964 
Table 1 Demographic characteristics and MMSE score of the two subsamples [i.e., the normal control group who remained healthy (NC-NC) and the initially healthy participants who progressed to AD dementia (NC-AD)]

\begin{tabular}{|c|c|c|c|c|}
\hline & $\mathrm{NC}-\mathrm{NC}^{\mathrm{a}}(n=26)$ & $\mathrm{NC}-\mathrm{AD}^{\mathrm{b}}(n=26)$ & $T / \chi^{2}$ & $p$ value \\
\hline Age $\pm \mathrm{SD}^{\mathrm{c}}$ (years) & $72.3 \pm 5.4$ & $72.8 \pm 4.6$ & 0.33 & 0.74 \\
\hline Education $\pm \mathrm{SD}$ (years) & $12.8 \pm 2.8$ & $12.6 \pm 2.7$ & -0.20 & 0.84 \\
\hline \%women & 42.3 & 42.3 & $0.00^{\mathrm{d}}$ & 1.00 \\
\hline $\mathrm{MMSE}^{\mathrm{e}} \pm \mathrm{SD}$ & $29.1 \pm 1.0$ & $28.7 \pm 1.4$ & -1.15 & 0.26 \\
\hline Observation time \pm SD (years) & $10.8 \pm 3.03$ & $7.8 \pm 2.7$ & -3.74 & $<0.001$ \\
\hline
\end{tabular}

${ }^{a} \mathrm{NC}-\mathrm{NC}=$ cognitively healthy participants who remained healthy

b $\mathrm{NC}-\mathrm{AD}=$ initially healthy participants who progressed to Alzheimer's disease dementia

c $S D$ standard deviation

d $\chi^{2}$ test

e MMSE Mini-mental state examination [29]

Declaration of Helsinki and its later amendments. All participants gave written informed consent.

\section{Neuropsychological measures}

All participants were administered the German version of the CERAD-NAB [21, 22]. This battery included seven subtests measuring verbal episodic learning (WordlistEncoding), verbal episodic memory (Wordlist-Delayed recall and Recognition), constructional praxis (FiguresCopy), visual episodic memory (Figures-Delayed recall), executive functions (verbal fluency), and language (Boston Naming Test, 15-items). Additionally, we computed three variables: a measure reflecting the proportion of correct words recalled during verbal delayed recall relative to the words recalled at word list learning trial 3 (Wordlist-Savings), a measure representing the total number of intrusions at Wordlist-Encoding and Wordlist-Delayed recall (Wordlist-Intrusion errors), and a measure describing the proportion of correctly reproduced figures at Figures-Delayed Recall relative to Figures-Copy (Figures-Savings). A description of these tests is provided in Table 2. Altogether, ten raw scores and demographically adjusted for age, gender, and education $z$-scores were derived (see $[25,33]$ ). Because education is a strong predictor for premorbid cognitive performance, the number of years of education was used as its surrogate [13, 34, 35]. A summary of the overall neuropsychological CERAD-NAB test performance is provided in Table S1 in the electronic Supplementary Materials (see Online Resource 1).

\section{Analyses}

Pearson product moment correlations were performed on the $z$-scores of all ten CERAD-NAB variables to illustrate subtest intercorrelations. The prevalence of CERAD-NAB low scores were calculated from the overall normative sample and both subsamples for six different cut-off scores that are frequently applied in clinical practice (see, e.g., [13]). The cut-off scores are listed below.

1. 1 st percentile $(z$-score $\leq-2.32)$.

2. 2.5th percentile $(z$-score $\leq-1.96)$.

3. 7th percentile $(z$-score $\leq-1.48)$.

4. 10th percentile $(z$-score $\leq-1.28)$.

5. 16th percentile $(z$-score $\leq-1.00)$.

6. 25 th percentile $(z$-score $\leq-0.67)$.

Because memory impairment alone is sufficient for a diagnosis of amnestic MCI [9], an additional analysis was conducted focusing only on the CERAD-NAB memory domains. Thus, the prevalence of low CERAD-NAB scores in the verbal and visual memory was calculated: WordlistEncoding, Wordlist-Delayed recall, Wordlist-Discriminability, Wordlist-Savings, Wordlist-Intrusion errors, Figures-Delayed recall, and Figures-Savings. These results are reported in the electronic Supplementary Material section (see Online Resource 2). To estimate the variability of the number of low scores in the 10 CERAD-NAB variables as well as to obtain the $95 \%$ confidence intervals (CI), we computed 1,000 bootstrap replicates [36].

In order to aid clinicians in daily practice and to facilitate clinical decision making (i.e., to determine whether a certain cognitive profile should be considered as normal or impaired), we aimed to provide a summary figure with the exact percentages of healthy older participants who obtain a certain number of low scores at or below each of the cutoff scores. Because cognitive impairment (due to any reason) is variably defined in clinical practice, we determined that probable cognitive impairment may be assumed, when $<10 \%$ of healthy older adults obtain a certain number of low scores below a given cut-off score (see also [1, 12]). That is, if the number of scores below a certain cut-off score was obtained by approximately $10 \%$ or less participants 
Table 2 Description of neuropsychological subtests of the CERAD-NAB ${ }^{\mathrm{a}}[21,22]$ used in this study

\begin{tabular}{|c|c|c|}
\hline Test variable & Test description & Function \\
\hline CERAD-NAB ${ }^{\text {a }}$ Wordlist-Encoding & $\begin{array}{l}\text { Total number of correctly learned words across three learning trials } \\
\text { (number of words per trial }=10 \text { ) }\end{array}$ & Verbal episodic learning \\
\hline CERAD-NAB ${ }^{\text {a }}$ Wordlist-Delayed recall & Total number of correctly remembered words after Wordlist-Encoding & Verbal episodic memory \\
\hline CERAD-NAB ${ }^{\text {a }}$ Wordlist-Savings & $\begin{array}{l}\text { Proportion correct words recalled during Wordlist-Delayed recall relative } \\
\text { to words learned at Wordlist-Encoding learning trial } 3\end{array}$ & Verbal episodic memory \\
\hline CERAD-NAB ${ }^{\text {a }}$ Wordlist-Discriminability & Percent of correctly recognized words from Wordlist-Encoding & Verbal episodic memory \\
\hline CERAD-NAB ${ }^{\mathrm{a}}$ Wordlist-Intrusion errors & $\begin{array}{l}\text { Total number of intrusions at Wordlist-Encoding and Wordlist-Delayed } \\
\text { recall }\end{array}$ & Executive functions \\
\hline CERAD-NAB ${ }^{\text {a }}$ Figures-Copy & Copy of four figures (circle, diamond, overlapping rectangles, cube) & Constructional praxis \\
\hline CERAD-NAB ${ }^{\text {a }}$ Figures-Delayed recall & Recall of figures reproduced at Figures-Copy & Visual episodic memory \\
\hline CERAD-NAB ${ }^{\text {a }}$ Figures-Savings & $\begin{array}{l}\text { Proportion correctly reproduced figures at Figures-Delayed recall relative } \\
\text { to Figures-Copy }\end{array}$ & Visual episodic memory \\
\hline Verbal fluency-Animals & Number of animals reproduced within $1 \mathrm{~min}$ & Executive functions \\
\hline $\mathrm{BNT}^{\mathrm{b}}(15$-items $)$ & Spontaneous naming of 15 black and white line drawings & Language \\
\hline
\end{tabular}

of the normative sample, we labeled this performance as probable cognitive impairment. Of course, any other cutoff (e.g., 5th percentile) could be used depending on the need of the examiner (e.g., high sensitivity vs. high specificity; the $10 \%$ cutoff used here serves as an example).

To examine whether this critical $10 \%$ border line may detect very early and subtle signs of cognitive impairment for each of the six cut-off scores, we compared baseline neuropsychological performance of 26 healthy participants who progressed to $\mathrm{AD}$ dementia (NC-AD) years later and their matched control group (NC-NC). Two-sided Fisher's exact tests were performed to determine baseline differences (i.e., at a healthy stage) in the subgroups, using an alpha level of $p<0.05$. All statistical analyses were performed with SPSS version 21 (SPSS Inc. IBM company, 2012).

\section{Results}

The correlation analysis between the $z$-scores of the ten CERAD-NAB tests revealed a pattern of coefficients that were mostly small to medium, indicating minimal to modest multicollinearity. Nearly all the correlations were smaller than 0.35 . The largest correlations were as follows: Figures-Delayed Recall and Figures-Savings $=0.89$, Wordlist-Delayed Recall and Wordlist-Savings $=0.76$, Wordlist-Delayed Recall and WordlistEncoding $=0.648$, and Wordlist-Delayed Recall and Wordlist-Recognition $=0.47$.

Figure 1 shows the cumulative percentages of healthy individuals who have a specific number of low test scores at or below each of the six cut-off scores. Because the percentage of participants who obtain seven or more low scores was very small (i.e., $\leq 7.5 \%$ ) even for the less stringent cut-off scores, Fig. 1 only illustrates the percentage of participants who obtained up to six low scores (i.e., $\geq 1$, $\geq 2, \geq 3, \geq 4, \geq 5, \geq 6$ ). These data show that a high percentage of the normative sample obtains at least one or more low scores at most cut-off scores and that the number of low scores varies as a function of the cut-off score.

In addition to the exact percentage of healthy individuals who obtain a certain number of low scores at or below each cut-off score, Fig. 2 provides information about the number of low scores required to determine probable cognitive impairment as well as the $95 \%$ CI calculated by using the bootstrap method [36]. Given our definition, we set the critical border, where approximately $10 \%$ of all participants obtain a certain number of low scores (see Fig. 2; white area). This $10 \%$ border serves as a critical threshold to differentiate between broadly normal numbers of low scores (see Fig. 2, light gray area) and an ambiguous area representing higher uncertainty about the diagnostic accuracy (see Fig. 2, dark gray area), i.e., participants whose number of low scores is situated in the light gray area are likely to be diagnosed as cognitively healthy, because a high percentage of the normative sample obtained a similar number of low scores, whereas the cognitive status of individuals whose number of low scores falls above the border in the dark gray area may be considered as abnormal, because only a small number (at most $\leq 7.5 \%$ at 25 th percentile, see Fig. 2 last column) of healthy older adults obtain such a high number of low scores. Thus, according to Fig. 2, when using the $10 \%$ border as the critical threshold, probable cognitive impairment across all 10 scores would be based on obtaining one or more low scores $\leq 1$ st percentile 


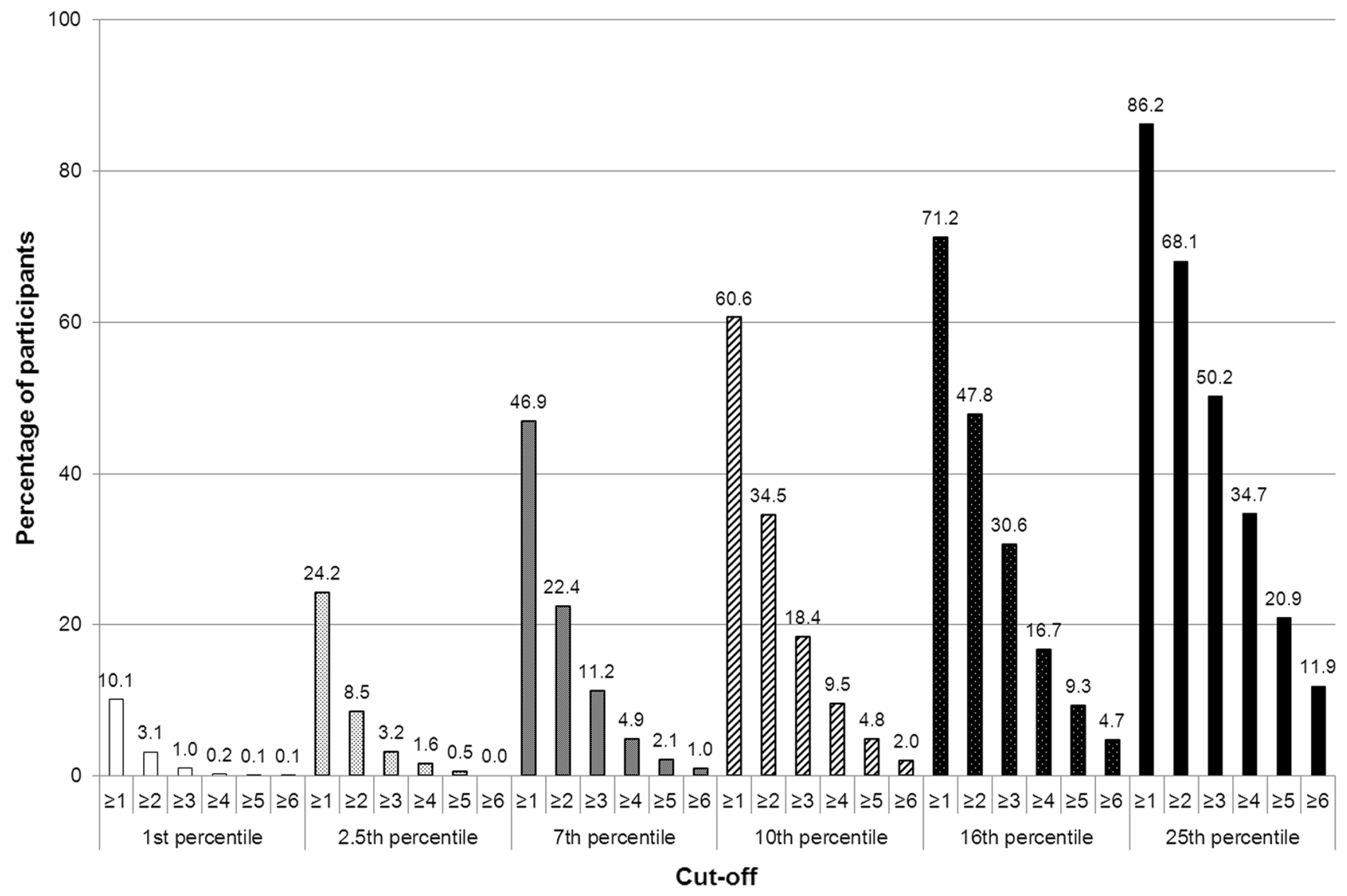

Fig. 1 Cumulative percentages of healthy older participants with a particular minimum number of low scores in the CERAD-NAB for six different cut-off scores

$(z \leq-2.32$; obtained by $10.1 \%$ of the normative sample), two or more low scores $\leq 2.5$ th percentile $(z \leq-1.96$; obtained by $8.5 \%$ of the normative sample), three or more low scores $\leq 7$ th percentile $(z \leq-1.48$; obtained by $11.2 \%$ of the normative sample), four or more low scores $\leq 10$ th percentile $(z \leq-1.28$; obtained by $9.5 \%$ of the normative sample), five or more low scores $\leq 16$ th percentile $(z \leq-1.00$; obtained by $9.3 \%$ of the normative sample), or six or more low scores $\leq 25$ th percentile $(z \leq-0.67$; obtained by $11.9 \%$ of the normative sample). The results of the separate analysis with the verbal and visual episodic memory domain only are reported in the electronic Supplementary Material section (see Online Resource 2).

Figure 3 illustrates the percentage of the $\mathrm{NC}-\mathrm{NC}$ and $\mathrm{NC}-\mathrm{AD}$ groups situated in the critical area beneath the $10 \%$ border (see Fig. 2) for each cut-off score at baseline examination. Consistently, more NC-AD participants are situated in the critical dark gray area compared to NC-NC participants irrespective of the used cut-off score (see Fig. 3; Table 3). Two-sided Fisher's exact tests were performed to examine potential baseline differences of the $\mathrm{NC}-\mathrm{NC}$ and $\mathrm{NC}-\mathrm{AD}$ groups. These results indicate only differences for less stringent cutoffs (i.e., 25th and 16th percentile) and a statistical trend toward differences in participants who later progressed to AD dementia to be located in the critical dark gray area compared to individuals who remained healthy, for the 10th percentile (see Table 3 ). It has to be noted that the OR for the 16th percentile could not be calculated because the denominator was zero. For purposes of estimation, we added the value 0.5 to each cell in the contingency table. The results of the separate analysis with the verbal and visual episodic memory domain only are reported in the electronic Supplementary Material section (see Online Resource 2).

The final set of analyses compared a simultaneous application of all impairment-criterion cut-off scores across groups. That is, we computed the base rate in the normative sample of meeting one or more criteria for probable cognitive impairment across the ten scores when all six cut-off scores are applied simultaneously. In the entire normative sample, $22 \%$ met criteria for cognitive impairment based on meeting one or more of the criteria in the white area in Fig. 2 (i.e., this is the percentage of people who meet criterion when all criteria are considered). In the subsample of 26 NC-NC participants, $19 \%$ met any of the criteria, whereas 


\begin{tabular}{|c|c|c|c|c|c|c|c|c|c|c|c|c|}
\hline \multicolumn{13}{|c|}{ Normative cut-off scores } \\
\hline & \multicolumn{2}{|c|}{$\begin{aligned} & \leq 1 \% \\
(z & \leq-2.32)\end{aligned}$} & \multicolumn{2}{|c|}{$\begin{array}{c}\leq 2.5 \% \\
(z \leq-1.96)\end{array}$} & \multicolumn{2}{|c|}{$\begin{aligned} & \leq 7 \% \\
(z & \leq-1.48)\end{aligned}$} & \multicolumn{2}{|c|}{$\begin{array}{c}\leq 10 \% \\
(z \leq-1.28)\end{array}$} & \multicolumn{2}{|c|}{$\begin{array}{c}\leq 16 \% \\
(z \leq-1.00)\end{array}$} & \multicolumn{2}{|c|}{$\begin{array}{c}\leq 25 \% \\
(z \leq-0.67)\end{array}$} \\
\hline $\begin{array}{c}\text { scores } \\
\leq \\
\text { cut-off }\end{array}$ & $\%[\mathrm{Cl}]$ & $\mathrm{cp}$ & $\%[\mathrm{Cl}]$ & $\mathrm{cp}$ & $\%[C l]$ & $\mathrm{cp}$ & $\%[\mathrm{Cl}]$ & $\mathrm{cp}$ & $\%[C \mid]$ & $\mathrm{cp}$ & $\%[\mathrm{Cl}]$ & $\mathrm{cp}$ \\
\hline 10 & 0.0 & 0.0 & 0.0 & 0.0 & 0.0 & 0.0 & 0.0 & 0.0 & 0.0 & 0.0 & $\begin{array}{c}0.1 \\
{[0.0-0.3]}\end{array}$ & 0.1 \\
\hline 9 & 0.0 & 0.0 & 0.0 & 0.0 & 0.0 & 0.0 & $\begin{array}{c}0.1 \\
{[0.0-0.3]}\end{array}$ & 0.1 & $\begin{array}{c}0.4 \\
{[0.1-0.7]}\end{array}$ & 0.4 & $\begin{array}{c}1.2 \\
{[0.6-1.9]}\end{array}$ & 1.3 \\
\hline 8 & 0.0 & 0.0 & $\begin{array}{c}0.1 \\
{[0.0-0.3]}\end{array}$ & 0.1 & $\begin{array}{c}0.2 \\
{[0.0-0.5]}\end{array}$ & 0.2 & $\begin{array}{c}0.1 \\
{[0.0-0.3]}\end{array}$ & 0.2 & $\begin{array}{c}0.7 \\
{[0.3-1.3]}\end{array}$ & 1.1 & $\begin{array}{c}2.0 \\
{[1.3-3.0]}\end{array}$ & 3.3 \\
\hline 7 & 0.0 & 0.0 & 0.0 & 0.1 & $\begin{array}{c}0.2 \\
{[0.0-0.5]}\end{array}$ & 0.4 & $\begin{array}{c}0.6 \\
{[0.2-1.0]}\end{array}$ & 0.7 & $\begin{array}{c}1.0 \\
{[0.5-1.7]}\end{array}$ & 2.1 & $\begin{array}{c}4.2 \\
{[3.0-5.4]}\end{array}$ & 7.5 \\
\hline 6 & $\begin{array}{c}0.1 \\
{[0.0-0.3]}\end{array}$ & 0.1 & 0.0 & 0.1 & $\begin{array}{c}0.6 \\
{[0.2-1.2]}\end{array}$ & 1.0 & $\begin{array}{c}1.3 \\
{[0.6-2.0]}\end{array}$ & 2.0 & $\begin{array}{c}2.6 \\
{[1.7-3.7]}\end{array}$ & 4.7 & $\begin{array}{c}4.4 \\
{[3.2-5.7]}\end{array}$ & 11.9 \\
\hline 5 & 0.0 & 0.1 & $\begin{array}{c}0.4 \\
{[0.1-0.8]}\end{array}$ & 0.5 & $\begin{array}{c}1.1 \\
{[0.6-1.8]}\end{array}$ & 2.1 & $\begin{array}{c}2.8 \\
{[1.8-3.8]}\end{array}$ & 4.8 & $\begin{array}{c}4.6 \\
{[3.4-5.8]}\end{array}$ & 9.3 & $\begin{array}{c}9.0 \\
{[7.1-10.6]}\end{array}$ & 20.9 \\
\hline 4 & $\begin{array}{c}0.1 \\
{[0.0-0.3]}\end{array}$ & 0.2 & $\begin{array}{c}1.1 \\
{[0.6-1.8]}\end{array}$ & 1.6 & $\begin{array}{c}2.8 \\
{[1.9-3.8]}\end{array}$ & 4.9 & $\begin{array}{c}4.7 \\
{[3.5-5.9]}\end{array}$ & 9.5 & $\begin{array}{c}7.3 \\
{[5.8-8.9]}\end{array}$ & 16.7 & $\begin{array}{c}13.8 \\
{[11.6-15.8]}\end{array}$ & 34.7 \\
\hline 3 & $\begin{array}{c}0.8 \\
{[0.4-1.4]}\end{array}$ & 1.0 & $\begin{array}{c}1.7 \\
{[0.9-2.4]}\end{array}$ & 3.2 & $\begin{array}{c}6.3 \\
{[4.9-7.8]}\end{array}$ & 11.2 & $\begin{array}{c}8.9 \\
{[7.2-10.6]}\end{array}$ & 18.4 & $\begin{array}{c}14.0 \\
{[12.0-16.1]}\end{array}$ & 30.6 & $\begin{array}{c}15.5 \\
{[13.4-17.9]}\end{array}$ & 50.2 \\
\hline 2 & $\begin{array}{l}2.1 \\
{[1.3-3.0]}\end{array}$ & 3.1 & $\begin{array}{c}5.3 \\
{[4.0-6.6]}\end{array}$ & 8.5 & $\begin{array}{c}11.2 \\
{[9.3-13.0]}\end{array}$ & 22.4 & $\begin{array}{c}16.1 \\
{[13.8-18.4]}\end{array}$ & 34.5 & $\begin{array}{c}17.2 \\
{[15.0-19.5]}\end{array}$ & 47.8 & $\begin{array}{c}17.9 \\
{[15.4-20.0]}\end{array}$ & 68.1 \\
\hline 1 & $\begin{array}{c}6.9 \\
{[5.5-8.5]}\end{array}$ & 10.1 & $\begin{array}{c}15.7 \\
{[13.4-18.0]}\end{array}$ & 24.2 & $\begin{array}{c}24.5 \\
{[22.0-27.2]}\end{array}$ & 46.9 & $\begin{array}{c}26.1 \\
{[23.3-28.5]}\end{array}$ & 60.6 & $\begin{array}{c}23.4 \\
{[21.0-25.9]}\end{array}$ & 71.2 & $\begin{array}{c}18.1 \\
{[15.9-20.6]}\end{array}$ & 86.2 \\
\hline 0 & $\begin{array}{c}89.9 \\
{[88.1-91.7]}\end{array}$ & 100.0 & $\begin{array}{c}75.8 \\
{[73.0-78.5]}\end{array}$ & 100.0 & $\begin{array}{c}53.1 \\
{[50.1-56.2]}\end{array}$ & 100.0 & $\begin{array}{c}39.4 \\
{[36.7-42.5]}\end{array}$ & 100.0 & $\begin{array}{c}28.8 \\
{[26.2-31.5]}\end{array}$ & 100.0 & $\begin{array}{c}13.8 \\
{[11.7-15.9]}\end{array}$ & 100.0 \\
\hline
\end{tabular}

Fig. 2 Base rates (in \%) of demographically adjusted low z-scores out of ten CERAD-NAB variables (far left column) for six different cut-off scores (second row from the top). CI $95 \%$ confidence interval, $c p$ cumulative percentage. The white area represents a critical border where circa $10 \%$ of all participants $(N=1,081)$ obtain a certain number of low scores and serves a threshold to differenti-

$54 \%$ of the $26 \mathrm{NC}-\mathrm{AD}$ participants met criteria for cognitive impairment based on meeting one or more of the criteria of the criteria in the white area in Fig. 2. This difference is statistically significant $[p=0.02, \mathrm{OR}=4.9,95 \%$ CI $(1.41$, 16.99)]. The same analysis was conducted for the memory domain only, and results are reported in the electronic Supplementary Material section (see Online Resource 2). Moreover, in the electronic Supplementary Material section, we also provide additional variations of combined criteria and their corresponding base rates (see Online Resource 3).

\section{Discussion}

This study provides information about the base rates of low scores in the CERAD-NAB in a normative sample of older ate between low (light gray area) and high (dark gray area) probabilities of pathological performance. Thus, neuropsychological results located in the light gray area would be interpreted as within normal limits, whereas results in the dark gray area would be interpreted as probable cognitive impairment

adults using six different cut-off scores commonly applied in clinical practice and research. The summary figure (Fig. 2) may be used as an important supplement in clinical practice when assessing cognitive performance and aims to reduce diagnostic errors in clinical decision making. Our main results support a number of studies demonstrating that a substantial percentage of healthy children [37-39], healthy adults $[40,41]$, and healthy older adults $[1,3,5$, 15] will obtain scores that fall within abnormal ranges when multiple tests are administered.

According to the Gaussian distribution, the number of low scores varies as a function of the cut-off score-the higher the cut-off score, the more abnormal test scores are required to diagnose cognitive impairment. However, the use of a certain cut-off score critically relates to the sensitivity and specificity of a test. For example, using a 


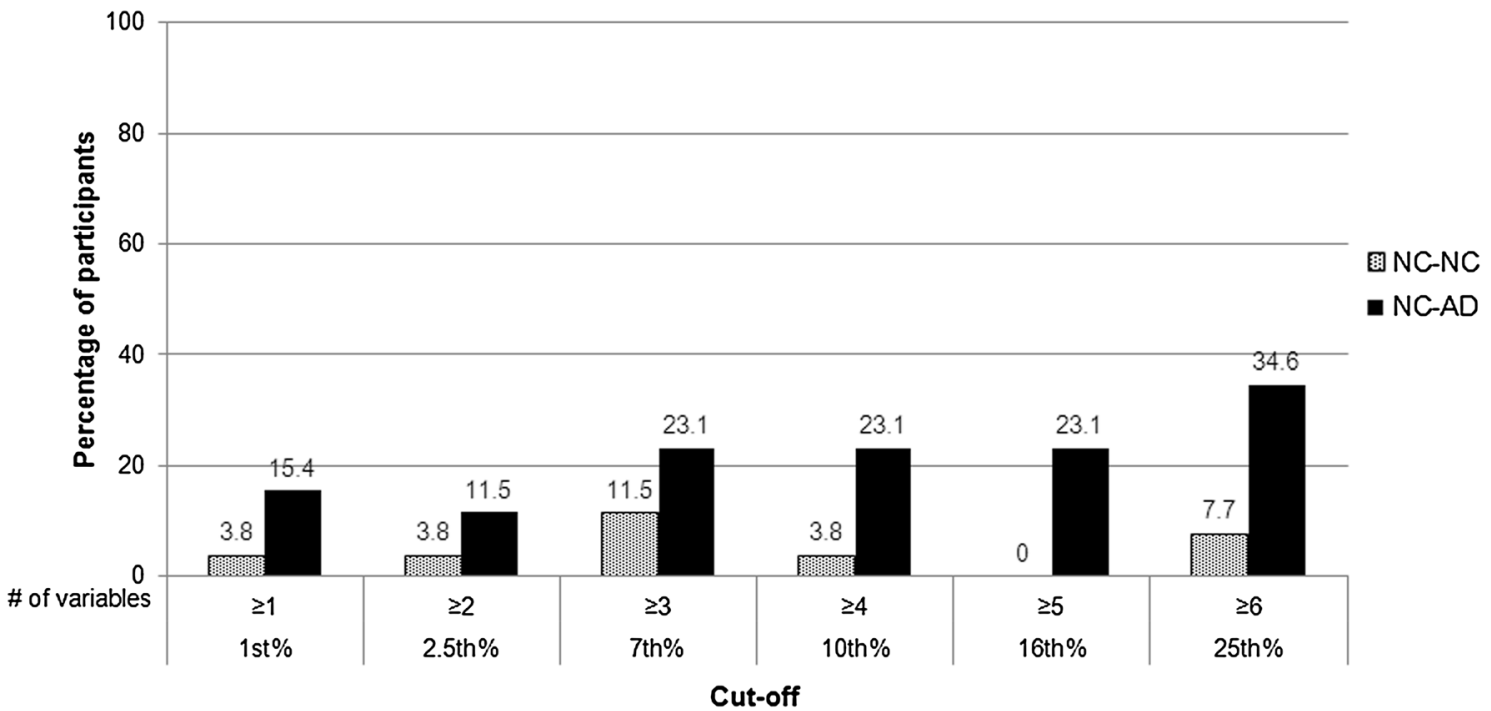

Fig. 3 Percentage of normal controls who remained normal (NCNC; $n=26$ ) and of initially healthy participants who later obtained a diagnosis of $\mathrm{AD}$ dementia $(\mathrm{NC}-\mathrm{AD} ; n=26)$ situated in the critical dark gray area beneath the $10 \%$ border (see Fig. 2) at each cutoff ( $x$-axis) at baseline

Table 3 Comparison of percentages of participants $\left(\mathrm{NC}-\mathrm{NC}^{\mathrm{a}}, \mathrm{NC}-\mathrm{AD}^{\mathrm{b}}\right)$ situated in the dark gray area in Fig. 2 (at baseline)

\begin{tabular}{|c|c|c|c|c|}
\hline & \multicolumn{2}{|c|}{$\%$ in the dark gray area ${ }^{c}$} & \multirow[t]{2}{*}{$p$ value $^{\mathrm{d}}$} & \multirow[t]{2}{*}{$\mathrm{OR}^{\mathrm{e}}\left(95 \% \mathrm{CI}^{\mathrm{f}}\right)$} \\
\hline & $\mathrm{NC}-\mathrm{NC}^{\mathrm{a}}(\%)$ & $\mathrm{NC}-\mathrm{AD}^{\mathrm{b}}(\%)$ & & \\
\hline 25 th percentile & 7.7 & 34.6 & $0.04 *$ & $6.35(1.22,33.19)$ \\
\hline 16 th percentile & 0 & 23.1 & $0.02 *$ & $16.8(0.90,315.89)$ \\
\hline 10th percentile & 3.8 & 23.1 & 0.10 & $7.5(0.83,67.49)$ \\
\hline 7th percentile & 11.5 & 23.1 & 0.47 & $2.3(0.51,10.41)$ \\
\hline 2.5th percentile & 3.8 & 11.5 & 0.61 & $3.3(0.32,33.62)$ \\
\hline 1st percentile & 3.8 & 15.4 & 0.35 & $4.6(0.47,43.78)$ \\
\hline
\end{tabular}

$* p$ value $<0.05$

${ }^{a} \mathrm{NC}-\mathrm{NC}=$ cognitively healthy participants who remained healthy

b $\mathrm{NC}-\mathrm{AD}=$ initially healthy participants who progressed to Alzheimer's disease dementia

${ }^{c}$ Dark gray area $=$ to be considered as a pathological result (see Fig. 2)

d Fisher's $p$ value tested by Fisher's exact test

e $O R$ odds ratio

${ }^{\mathrm{f}} \mathrm{CI}$ confidence interval

less stringent criterion for impairment [e.g., the 25th percentile $(z$-score $\leq-0.67)$ ] indeed increases the identification of true impairment (increased sensitivity), but it is also associated with reduced specificity (i.e., the percentage of healthy individuals erroneously being classified as impaired is high; see Fig. 1) [12]. Moreover, administering more than one neuropsychological test, which is mandatory to obtain a comprehensive understanding of someone's cognitive performance, yields multiple test scores and inherently increases the probability of abnormal scores [12, 14, 15]. For example, Schretlen et al. [15] reported that when examining healthy adults' cognitive abilities by administering a test battery containing 10,25 or 43 subtests, 15, 40 and $57 \%$, respectively, of healthy adults obtain two or more scores below the 7 th percentile (i.e., $z$-score $=-1.5$ ). Moreover, choosing a more stringent criterion ( $z$-score $=-1.96)$, still 3,13 and $24 \%$, respectively, obtain two or more low scores.

Beside these methodological issues, possible explanations for low test performance among these healthy adults may be due to situational or personal factors such as motivational fluctuations, increased distractibility during 
testing, inattentiveness, and measurement error [20], or also longstanding personal weaknesses in certain areas or intraindividual cognitive variability across different cognitive domains [5, 42]. Another explanation of low scores in cognitively normal adults is the possibility of poor effort. However, as Binder et al. [20] mention in their reviewand this is very much in line with our own observationsparticipants taking part in research studies rarely exhibit insufficient effort, since their participation is voluntary. Moreover, systematic and random errors as additional sources for low scores were minimized as best as possible. Specifically, due to the large number of participants in this study, multiple examiners were needed to administer the tests. All examiners were thoroughly trained by experienced neuropsychologists, and instructions were given in a highly standardized way. In order to reduce any discomfort of participants, a warming-up chat was carried-out at the beginning of the examination, which took place in a quiet room without distractors. In addition, if necessary and appropriate, short breaks during the testing were allowed to avoid fatigue. To safeguard against errors at data entry, this procedure was triple-checked. Thus, these results emphasize that low scores do not necessarily need to be indicative of impairment, but may also represent normal variability of cognitive abilities within healthy adults. This is also suggested by a number of recent studies which illustrate that a considerable percentage of participants (up to $25 \%$ ) are diagnosed as cognitively impaired at baseline evaluation but revert to normal levels of functioning at follow-up examination [43-45], indicating diagnostic instability over time. Indeed, some of these misdiagnosed patients may have reverted due to adequate treatment of factors that may have caused cognitive impairment other than neurodegeneration (e.g., mood disorder, vascular risk factors). However, it can be assumed that this high number of individuals exhibiting improved or normal cognition at follow-up also contains a certain number of incorrectly diagnosed people at baseline because the probability of obtaining some low scores was not taken into account [44]. Interestingly, there is evidence that the likelihood to score within normal limits at follow-up is higher when the low scores at baseline were obtained in non-memory tests (i.e., non-amnestic MCI) $[44,45]$. These results indicate that, in addition to the pure number of variables with a low score, it is important to also consider the affected cognitive domain.

The present study, however, also illustrates that some of these "healthy" participants may not be healthy and might be at a higher risk to progress to $\mathrm{AD}$ (i.e., they are in a prodromal stage of dementia). For example, our findings show that six or more scores (out of ten) below the 25th percentile occurred in about $12 \%$ of the normative sample (see Fig. 2), in $8 \%$ of the 26 older adults who remained cognitively healthy over time, but in almost $35 \%$ of older adults who progressed to dementia (see Fig. 3). However, this result must be interpreted with caution because sample sizes were small. Nevertheless, the future dementia patients might have obtained low scores because they were already on the path of cognitive decline associated with future dementia. This thought corroborates with findings of current neuropsychological research investigating the preclinical stages of MCI and AD suggesting that low cognitive performance can be evidenced in a premorbid phase of later progressors to dementia more than a decade before diagnosis [7, 26, 46, 47]. For example, Amieva et al. [46] found in a sample of 350 preclinical AD patients and in demographically matched healthy controls that semantic and episodic memory declined 9-12 years prior to the diagnosis of $A D$ dementia [46]. In light of these considerations, using our summary figure (Fig. 2) may help to improve diagnostic decision making in clinical practice. An illustrative example for the application of these base rates can be found in the electronic Supplementary Materials section (see Online Resource 4).

This study has some limitations to consider. The sample used in this study was a convenience sample of a subsample of the original Basel study initiated in 1959, consisting predominantly of (former) employees of the pharmaceutical industry in Basel [48]. Thus, its representativeness is somewhat limited to rather better educated older individuals. Additionally, all CERAD-NAB variables exhibit a skewed distribution, comparable to most neuropsychological test variables. However, a close approximation to a normal distribution for all neuropsychological variables was accomplished by applying monotone transformations [25]. Because we aimed to improve diagnostic accuracy in a neuropsychological assessment and low cognitive performance is indicative of impairment, normally distributed scores are primarily needed for the diagnostically relevant lower tail, while a more liberal criterion for the upper tail does not influence diagnostic validity. Further, although all participants underwent a careful and comprehensive neuropsychological and medical screening, it is not known whether some of the participants in the normative study were in a prodromal stage of $\mathrm{AD}$, a condition that would mitigate the results. As illustrated in the subsample of 26 participants who later were diagnosed with dementia, we presume that some actually might have already been in a prodromal stage. The results from the baseline comparison between the $\mathrm{NC}-\mathrm{NC}$ and $\mathrm{NC}-\mathrm{AD}$ group are based on a very small sample and rather serve as a qualitative comparison. These results need to be studied in a larger sample. Moreover, we treated all CERAD-NAB variables as equally informative, although a high number of them were related to episodic memory.

Early and accurate diagnosis of MCI and AD represents a major and challenging goal of current neuropsychological 
research. The results of the present study substantially contribute to an improvement and facilitation of neuropsychological diagnostics and are highly relevant in clinical practice by illustrating that awareness of the base rates of low scores has important implications for the diagnosis of MCI or prodromal $\mathrm{AD}$. It is important to note that these base rate data are meant to only supplement clinical judgment, as neuropsychological test results need to be interpreted in conjunction with results of additional examinations (e.g., the patient's medical history and premorbid functioning (see also [19], informant-based and self-reported changes in the activities of daily living, structural magnetic resonance imaging, results cerebrospinal fluid analyses [49], etc.). Future research should investigate whether base rates of low scores in specific cognitive domains may have differential diagnostic and prognostic value. Additionally, as already mentioned, a broad range of cut-off scores is used to assess cognitive impairment. Future investigations with a large longitudinal sample might help to empirically define the optimal cut-off score(s) to discriminate between incipient neurocognitive disorder and normal aging. Moreover, because repeated neuropsychological testing is commonly used to evaluate decline over time, future research may apply the same methodology to determine the base rates of abnormal change of scores. By defining normal variability in change scores at subsequent evaluations, interpretation of follow-up results will be more valid.

Acknowledgments This work was supported by grants from the Swiss National Science Foundation (Grant No. 3200-049107 to A.U.M), from the Novartis Foundation, Basel (A.U.M.), Switzerland, and from GlaxoSmithKline (A.U.M.). We gratefully acknowledge the continuous enthusiasm of the participants of the BAsel Study on the ELderly project. A part of this paper has been presented as a poster at the Alzheimer's Association International Conference in Boston, MA, USA (July 13-18, 2013).

Conflict of interest All authors declare that they have no conflict of interest.

Open Access This article is distributed under the terms of the Creative Commons Attribution License which permits any use, distribution, and reproduction in any medium, provided the original author(s) and the source are credited.

\section{References}

1. Brooks BL, Iverson GL, Feldman HH, Holdnack JA (2009) Minimizing misdiagnosis: psychometric criteria for possible or probable memory impairment. Dement Geriatr Cogn Disord 27(5):439-450. doi:10.1159/000215390

2. Brooks BL, Iverson GL, Holdnack JA, Feldman HH (2008) Potential for misclassification of mild cognitive impairment: a study of memory scores on the Wechsler Memory Scale-III in healthy older adults. J Int Neuropsychol Soc 14(3):463-478. doi:10.1017/S1355617708080521
3. Brooks BL, Iverson GL, White T (2007) Substantial risk of "accidental MCI" in healthy older adults: base rates of low memory scores in neuropsychological assessment. J Int Neuropsychol Soc 13(3):490-500. doi:10.1017/S1355617707070531

4. Brooks BL, Iverson GL, White T (2009) Advanced interpretation of the neuropsychological assessment battery with older adults: base rate analyses, discrepancy scores, and interpreting change. Arch Clin Neuropsychol 24(7):647-657. doi:10.1093/arclin/acp061

5. Gunner JH, Miele AS, Lynch JK, McCaffrey RJ (2012) Performance of non-neurological older adults on the Wisconsin Card Sorting Test and the Stroop Color-Word Test: Normal variability or cognitive impairment? Arch Clin Neuropsychol 27(4):398405. doi:10.1093/arclin/acs047

6. Palmer BW, Boone KB, Lesser IM, Wohl MA (1998) Base rates of "impaired" neuropsychological test performance among healthy older adults. Arch Clin Neuropsychol 13(6):503-511. doi:10.1016/S0887-6177(97)00037-1

7. Amieva H, Jacqmin-Gadda $\mathrm{H}$, Orgogozo J-M, Le Carret $\mathrm{N}$, Helmer C, Letenneur L et al (2005) The 9 year cognitive decline before dementia of the Alzheimer type: a prospective populationbased study. Brain 128(5):1093-1101. doi:10.1093/brain/awh451

8. Sperling RA, Aisen PS, Beckett LA, Bennett DA, Craft SC, Fagan AM et al (2011) Toward defining the preclinical stages of Alzheimer's disease: recommendations from the National Institute on Aging-Alzheimer's Association workgroups on diagnostic guidelines for Alzheimer's disease. Alzheimers Dement 7(3):280-292. doi:10.1016/j.jalz.2011.03.003

9. Winblad B, Palmer K, Kivipelto M, Jelic V, Fratiglioni L, Wahlund L-O et al (2004) Mild cognitive impairment—beyond controversies, towards a consensus: report of the International Working Group on Mild Cognitive Impairment. J Intern Med 256(3):240246. doi:10.1111/j.1365-2796.2004.01380.x

10. Hedden T, Gabrieli JDE (2004) Insights into the ageing mind: a view from cognitive neuroscience. Nat Rev Neurosci 5(2):87-96. doi:10.1038/nrn1323

11. Salthouse $T$ (2010) Influence of age on practice effects in longitudinal neurocognitive change. Neuropsychology 24(5):563-572. doi:10.1037/a0019026

12. Iverson GL, Brooks BL (2011) Improving accuracy for identifying cognitive impairment. In: Schoenberg MR, Scott JG (eds) The little black book of neuropsychology: a syndrome based approach. Springer, New York, pp 923-950

13. Lezak MD, Howieson DB, Bigler ED, Tranel D (2012) Neuropsychological assessment, 5th edn. Oxford University Press, New York

14. Crawford JR, Garthwaite PH, Gault CB (2007) Estimating the percentage of the population with abnormally low scores (or abnormally large score differences) on standardized neuropsychological test batteries: a generic method with applications. Neuropsychology 21(4):419-430. doi:10.1037/0894-4105.21.4.419

15. Schretlen DJ, Testa SM, Winicki JM, Pearlson GD, Gordon B (2008) Frequency and bases of abnormal performance by healthy adults on neuropsychological testing. J Int Neuropsychol Soc 14(03):436-445. doi:10.1017/S1355617708080387

16. Petersen RC, Smith GE, Waring SC, Ivnik RJ, Tangalos EG, Kokmen E (1999) Mild cognitive impairment: clinical characterization and outcome. Arch Neurol 56(3):303-308

17. Albert MS, DeKosky ST, Dickson D, Dubois B, Feldman HH, Fox NC et al (2011) The diagnosis of mild cognitive impairment due to Alzheimer's disease: recommendations from the National Institute on Aging-Alzheimer's Association workgroups on diagnostic guidelines for Alzheimer's disease. Alzheimers Dement 7(3):270-279. doi:10.1016/j.jalz.2011.03.008

18. American Psychiatric Association (2013) Diagnostic and statistical manual of mental disorders: DSM-5, 5th edn. American Psychiatric Association Press, Washington 
19. Ivnik RJ, Smith GE, Petersen RC, Boeve BF, Kokmen E, Tangalos EG (2000) Diagnostic accuracy of four approaches to interpreting neuropsychological test data. Neuropsychology 14(2):163-177

20. Binder LM, Iverson GL, Brooks BL (2009) To err is human: "Abnormal" neuropsychological scores and variability are common in healthy adults. Arch Clin Neuropsychol 24:31-46. doi:10.1093/arclin/acn001

21. Welsh K, Butters N, Mohs RC, Beekly D, Edland S, Fillenbaum $\mathrm{G}$ et al (1994) The Consortium to Establish a Registry for Alzheimer's Disease (CERAD). Part V. A normative study of the neuropsychological battery. Neurology 44(4):609. doi:10.1212/ WNL.44.4.609

22. Morris JC, Heyman A, Mohs RC, Hughes JP, van Belle G, Fillenbaum $G$ et al (1989) The consortium to establish a registry for Alzheimer's disease (CERAD). Part I. Clinical and neuropsychological assessment of Alzheimer's disease. Neurology 39(9):1159-1165

23. Beck IR, Schmid NS, Berres M, Monsch AU (2014) Establishing robust cognitive dimensions for characterization and differentiation of patients with Alzheimer's disease, mild cognitive impairment, frontotemporal dementia and depression. Int J Geriatr Psychiatry 29(6):624-634. doi:10.1002/gps.4045

24. Ehrensperger MM, Berres M, Taylor KI, Monsch AU (2010) Early detection of Alzheimer's disease with a total score of the German CERAD. J Int Neuropsychol Soc 16(5):910-920. doi:10.1017/S1355617710000822

25. Berres M, Zehnder A, Bläsi S, Monsch AU (2008) Evaluation of diagnostic scores with adjustment for covariates. Stat Med 27(10):1777-1790. doi:10.1002/sim.3120

26. Schmid NS, Taylor KI, Foldi NS, Berres M, Monsch AU (2013) Neuropsychological signs of Alzheimer's disease 8 years prior to diagnosis. J Alzheimers Dis 34(2):537-546. doi:10.3233/ JAD-121234

27. Zehnder AE, Bläsi S, Berres $M$, Monsch AU, Stähelin HB, Spiegel R (2009) Impact of APOE status on cognitive maintenance in healthy elderly persons. Int $\mathrm{J}$ Geriatr Psychiatry 24(2):132-141. doi:10.1002/gps.2080

28. Monsch AU, Thalmann B, Schneitter M, Bernasconi F, Aebi C, Camachova Z et al (2000) The Basel study on the elderly's search for preclinical cognitive markers of Alzheimer's disease. Neurobiol Aging 21:31

29. Folstein MF, Folstein SE, McHugh PR (1975) "Mini-mental state": a practical method for grading the cognitive state of patients for the clinician. J Psychiatr Res 12(3):189-198. doi:10.1016/0022-3956(75)90026-6

30. American Psychiatric Association (1994) Diagnostic and statistical manual of mental disorders: DSM-IV, 4th edn. American Psychiatric Association Press, Washington

31. McKhann G, Drachman D, Folstein M, Katzman R, Price D, Stadlan EM (1984) Clinical diagnosis of Alzheimer's disease report of the NINCDS-ADRDA Work Group under the auspices of Department of Health and Human Services Task Force on Alzheimer's Disease. Neurology 34(7):939. doi:10.1212/ WNL.34.7.939

32. Monsch AU, Kressig RW (2010) Specific care program for the older adults: memory clinics. Eur Geriatr Med 1(2):128-131. doi:10.1016/j.eurger.2010.03.006

33. Berres M, Monsch AU, Bernasconi F, Thalmann B, Stähelin HB (2000) Normal ranges of neuropsychological tests for the disease of Alzheimer's disease. Stud Health Technol Inform 77:195-199

34. Heaton RK, Taylor MJ, Manly J (2003) Demographic effects and use of demographically corrected norms with the WAIS-III and
WMS-III. In: Tulsky DS, Saklofske DH, Chalune GJ, Heaton RK, Ivnik RJ (eds) Clinical interpretation of the WAIS-III and WMS-III. Academic Press, San Diego, pp 181-210

35. Spreen O, Strauss E (1998) A compendium of neuropsychological tests, 2nd edn. Oxford University Press, New York

36. Efron B (1979) Bootstrap methods: another look at the jackknife. Ann Stat 7(1):1-26. doi:10.1214/aos/1176344552

37. Brooks BL, Iverson GL (2012) Improving accuracy when identifying cognitive impairment in pediatric neuropsychological assessments. In: Sherman EMS, Brooks BL (eds) Pediatric forensic neuropsychology. Oxford University Press, New York, pp 66-88

38. Brooks BL, Iverson GL, Koushik NS, Mazur-Mosiewicz A, McNeill HA, Reynolds CR (2013) Prevalence of low scores in children and adolescents on the test of verbal conceptualization and fluency. Appl Neuropsychol Child 2(1):70-77. doi:10.1080/2 1622965.2012.742792

39. Brooks BL, Iverson GL, Sherman EMS, Holdnack JA (2009) Healthy children and adolescents obtain some low scores across a battery of memory tests. J Int Neuropsychol Soc 15(04):613-617. doi:10.1017/S1355617709090651

40. Axelrod BN, Wall JR (2007) Expectancy of impaired neuropsychological test scores in a non-clinical sample. Int $\mathbf{J}$ Neurosci 117(11):1591-1602. doi:10.1080/00207450600941189

41. Brooks BL, Iverson GL, Lanting SC, Horton AM, Reynolds CR (2012) Improving test interpretation for detecting executive dysfunction in adults and older adults: prevalence of low scores on the test of verbal conceptualization and fluency. Appl Neuropsychol 19(1):61-70. doi:10.1080/09084282.2012.651951

42. Schretlen DJ, Munro CA, Anthony JC, Pearlson GD (2003) Examining the range of normal intraindividual variability in neuropsychological test performance. J Int Neuropsychol Soc 9(6):864-870. doi:10.1017/S1355617703960061

43. Klekociuk SZ, Summers JJ, Vickers JC, Summers MJ (2014) Reducing false positive diagnoses in mild cognitive impairment: the importance of comprehensive neuropsychological assessment. Eur J Neurol 21(10):1330-1336. doi:10.1111/ene.12488

44. Jak AJ, Bondi MW, Delano-Wood L, Wierenga C, Corey-Bloom J, Salmon DP et al (2009) Quantification of five neuropsychological approaches to defining mild cognitive impairment. Am Assoc Geriatr Psychiatry 17(5):368-375. doi:10.1097/ JGP.0b013e31819431d5

45. Loewenstein DA, Acevedo A, Agron J, Duara R (2007) Stability of neurocognitive impairment in different subtypes of mild cognitive impairment. Dement Geriatr Cogn Disord 23(2):82-86. doi:10.1159/000097304

46. Amieva H, Le Goff M, Millet X, Orgogozo J-M, Pérès K, Barberger-Gateau P et al (2008) Prodromal Alzheimer's disease: successive emergence of the clinical symptoms. Ann Neurol 64(5):492-498. doi:10.1002/ana.21509

47. Howieson DB, Carlson NE, Moore MM, Wasserman D, Abendroth CD, Payne-Murphy J et al (2008) Trajectory of mild cognitive impairment onset. J Int Neuropsychol Soc 14(02):192-198. doi:10.1017/S1355617708080375

48. Widmer LK, Stähelin HB, Nissen C, da Silva A (1981) Venen-, Arterien-Krankheiten, koronare Herzkrankheit bei BerufstätigenProspektiv-epidemiologische Untersuchung Basler Studie I-III 1959-1978. Hans Huber Verlag, Bern

49. De Leon MJ, Mosconi L, Blennow K, De Santis S, Zinkowski R, Mehta PD et al (2007) Imaging and CSF studies in the preclinical diagnosis of Alzheimer's disease. Ann N. Y. Acad Sci 1097(1):114-145. doi:10.1196/annals.1379.012 